\title{
Multifocal Simultaneous Remote Intracerebral Hemorrhage After t-PA for Ischemic Stroke
}

\author{
Gustavo Saposnik, Jacob Schneiderman, Neville Bayer, On behalf of the Stroke Outcome Research \\ Canada (SORCan) Working Group
}

Can. J. Neurol. Sci. 2008; 35: 519-521

Intracranial hemorrhage is the most feared complication of thrombolytic therapy for acute ischemic stroke. Its frequency in large trials varies from $6.6 \%$ to $8.8 \%, 1,2$ but has been decreased by following the current guidelines for the management of thrombolysis for acute stroke in prospective community studies. Multifocal intracerebral hemorrhage is a rare complication after thrombolysis. We herein describe two cases of multifocal simultaneous intracerebral hemorrhage (MSE-ICH) after receiving thrombolytic therapy for acute stroke.

\section{METHODS}

From Jan 2006 to July 2007 we analyzed all patients receiving thrombolytic therapy for acute stroke admitted to St. Michael's Hospital site participating in the Registry of the Canadian Stroke Network. Patients met the Canadian guidelines for Intravenous Thrombolytic Treatment in Acute Stroke. Imaging from patients with MSE-ICH after thrombolysis were reported by neuroradiologists who were blinded of the clinical status of the patients.

\section{RESULTS}

Among 319 patients admitted with an acute ischemic stroke, $70(21.9 \%)$ received iv thrombolysis. Five patients $(7.1 \%)$ developed a symptomatic intracerebral hemorrhage, while two (2.9\%) developed MSE-ICH within 24 hours (Figure 1A). Patient \#1 (90 years old) presented with Right-middle carotid artery (MCA) syndrome, while patient \#2 (71 years old) presented Left-MCA syndrome. National Institute of Health Stroke Scale (NIHSS) on admission were 15 and 17 respectively. Blood pressure required control with Labetalol pre-thrombolysis in both patients. There was no clinical history of coagulopathy or systemic bleeding in these patients. Routine blood work, repeated coagulation studies and fibrinogen were normal on both patients. Twenty-four hour computed tomogram (CT) head from both patients with MSE-ICH are shown in Figure 1A. Both patients died within 96 hours. A postmortem magnetic resonance image (MRI) was completed in Patient \#2 (Figure 1B).

\section{DISCUSSION}

Intracerebral hemorrhage (ICH) is one of the most feared complications after thrombolytic therapy for acute myocardial infarct or ischemic stroke. While most of the intracranial hemorrhages are confined to the ischemic territory, extraischemic multifocal intracerebral hematomas have been
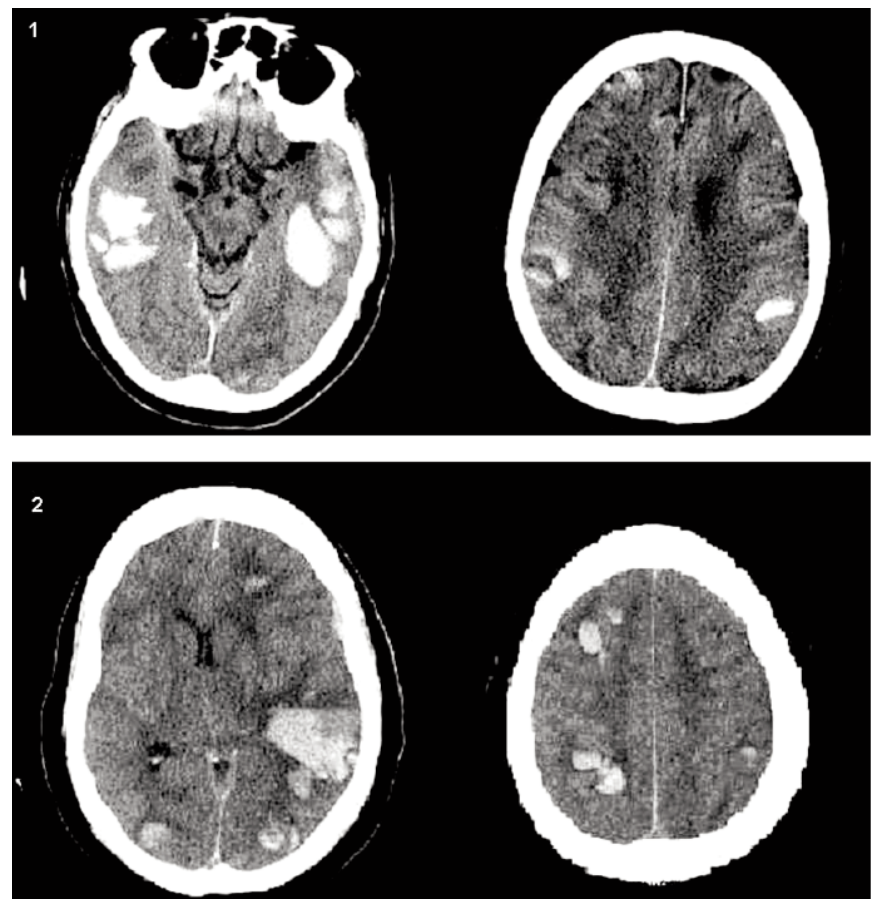

Figure 1A. Non contrast-CT shows extensive multifocal intraparenchymal hemorrhages. No visible hemorrhage within the brainstem and cerebellum. Intracerebral hemorrhages were also outside the area of the ischemic infarction (Right-MCA territory in patient \#1 and Left-MCA territory in patient \#2). 


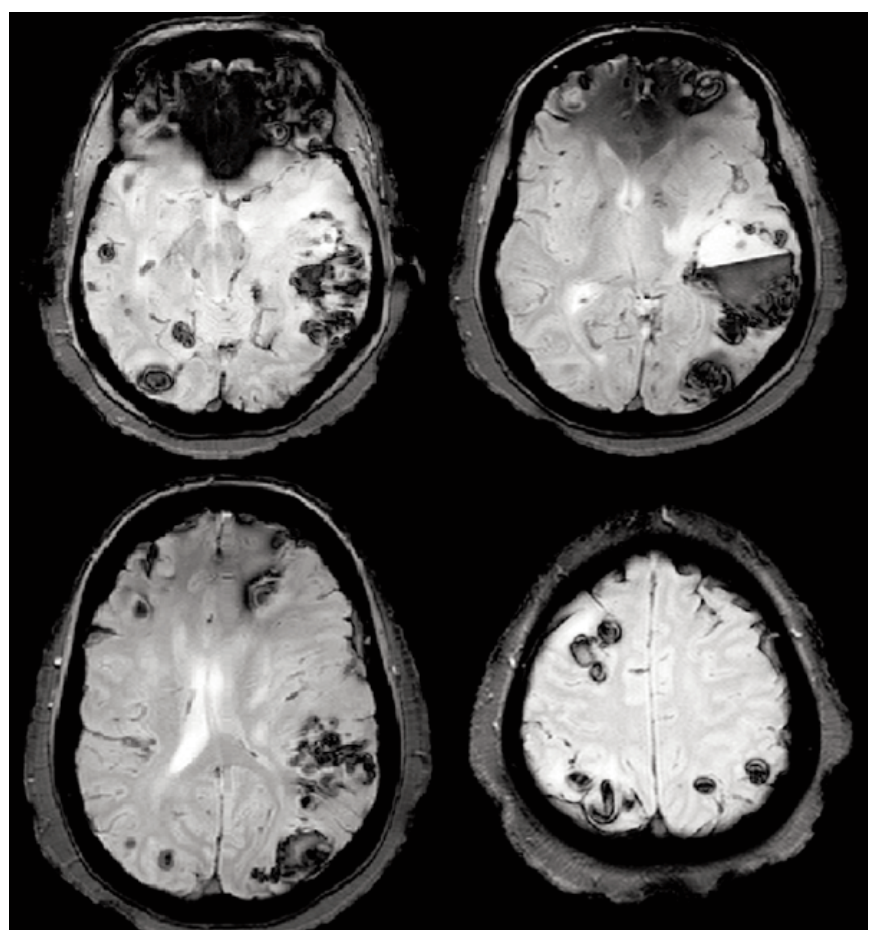

Figure 1B. Post-mortem MRI of the brain, Gradient echo sequence (Patient 2). Multiple bilateral acute intraparenchymal hematomas, several demonstrating blood levels. The largest intraparenchymal hematoma is in the posterior left parietal lobe measuring $49 \times 44 \times 31$ mm causing mass-effect, effacement of the left lateral ventricle, left uncal herniation, and compression of the midbrain.

described after thrombolysis for myocardial infarction, but less common after treatment for acute ischemic stroke. ${ }^{3,4}$

In the Global Utilization of Streptokinase and Tissue Plasminogen Activator for Occluded Coronary Arteries (GUSTO) I trial, 244 cases of symptomatic ICH were reported. ${ }^{4}$ Most hemorrhages were large, lobar (77\%), and intraparenchymal (82\%), and occurred within the first 24 hours. Fifteen percent $(36 / 244)$ were multifocal, and occurred significantly earlier than unifocal ICH after the administration of the thrombolytic agent $(\mathrm{p}=.002)$. The overall frequency of symptomatic intracranial hemorrhage in GUSTO-1 was $0.65 \%$. Similar findings were observed in large trials using intravenous Alteplase for acute stroke. In the National Institute of Neurological Diseases (NINDS) trial, 6.4\% of treated patients developed an ICH. The incidence of extra-ischemic intracranial hematomas (multifocal or not) occurred in 20\% (4/20) of all t-PA related symptomatic ICH and in 1.3\% (4/311) of all t-PA treated patients. ${ }^{1}$ In the European Cooperative Acute Stroke Study (ECASS) I and II studies, the incidence of brain hemorrhages in regions without visible ischemic tissue changes was 23 of 620 (3.7\%) and 16 of $800(2.0 \%))^{2,5}$ In the Lyon study, the multifocal form of extra-ischemic parenchymal hematomas overall represented $0.6 \%$, and $9 \%$ of all parenchymal hematomas. These studies provide no information on the possibility of a preexisting amyloid angiopathic in patients with multifocal hemorrhages.

General medical conditions have been associated with multiple and simultaneous intracranial bleeding including leukemia and other blood dyscrasias, increased fibrinogen degradation products (FDP), neoplasms, vasculitis, and venous sinus thrombosis. Although leukoaraiosis has been associated with an increase risk of bleeding post-thrombolysis, most of the intracranial hemorrhages are limited to the ischemic area. ${ }^{6}$ In the absence of coagulopathies, defibrinogenemia, arteriovenous malformations and cerebral amyloid angiopathy (CAA) have been described. ${ }^{7}$ Although hypertension appears to be a cofactor in the genesis of $\mathrm{ICH}$, it is unclear as to the possible additive effect of arterial hypertension during the acute phase on angiopathy linked to gradient echo positive microbleeds and CAA. $^{8}$

Magnetic resonance imaging (especially T2-weighted gradient echo and FLAIR sequences) may provide useful information by detecting microhemorrhages or microangiopathy changes which increase the risk of intracerebral bleeding. ${ }^{9}$

In a recent metanalysis, hypertension, diabetes and ischemic heart disease were associated with brain microbleeds. The authors also acknowledged several research limitations in the understanding of this medical condition. ${ }^{10}$

Animal models studying the expression of matrix metalloproteinases have provide some clues on the possible underlying mechanisms to explain the multifocal intracranial bleeding. Studies on transgenic mice with CAA features (APP23) revealed a particular sensitivity to develop hemorrhages after rt-PA thrombolysis. ${ }^{11,12}$

\section{Conclusion}

In conclusion, multifocal intracranial hemorrhage is uncommon, usually symptomatic, and occurs within the first 24 hours after the administration of thrombolytic therapy. The prognosis is usually poor. An MRI of the head including a gradient echo sequence may help determine remote hemorrhages associated with amyloid angiopathy.

\section{RESEARCH SUPPORT}

This research was supported in part by grants from Heart Stroke Foundation of Canada, Canadian Institutes for Health Research, Department of Research at St. Michael's Hospital and Connaught Foundation (University of Toronto) given to Dr. Gustavo Saposnik.

The investigators acted as the sponsors of the study. None of the supporting agencies had input on the design, access to the data, analyses, interpretation, or publication of the study.

\section{ACKNOWLEDGEMENTS}

The authors thank Drs Walter Montanera, Lynne Noel de Tilly and Thomas Marotta for their support. We appreciate the support of Li Ka Shing Knowledge Translation institute, Research Department and members of the Stroke Team at St. Michael's Hospital, Toronto (South Eastern Toronto Stroke Centre). 


\section{REFERENCES}

1. Intracerebral hemorrhage after intravenous t-PA therapy for ischemic stroke. The NINDS t-PA Stroke Study Group. Stroke. 1997;28:2109-18.

2. Fiorelli M, Bastianello S, von Kummer R, von Kummer R, del Zoppo GJ, Larrue V, et al. Hemorrhagic transformation within 36 hours of a cerebral infarct: relationships with early clinical deterioration and three-month outcome in the European Cooperative Acute Stroke Study I (ECASS I) cohort. Stroke. 1999;30:2280-4.

3. Sloan MA, Price TR, Petito CK, Randall AM, Solomon RE, Terrin ML, et al. Clinical features and pathogenesis of intracerebral hemorrhage after rt-PA and heparin therapy for acute myocardial infarction: the Thrombolysis in Myocardial Infarction (TIMI) II Pilot and Randomized Clinical Trial combined experience. Neurology. 1995;45:649-58.

4. Gebel JM, Sila CA, Sloan MA, Granger CB, Mahaffey KW, Weisenberger $J$, et al. Thrombolysis-related intracranial hemorrhage: a radiographic analysis of 244 cases from the GUSTO-1 trial with clinical correlation. Global Utilization of Streptokinase and Tissue Plasminogen Activator for Occluded Coronary Arteries. Stroke. 1998;29:563-9.

5. Trouillas P, Derex L, Honnorat J, Philippeau F, Turjman F, Hermier M, et al. Final results of the Lyon Protocol (200 Cases): Effect of intravenous rtPA within 7 hours without radiological and clinical exclusions in carotid artery territory acute cerebral infarcts. Stroke. 2003;34:284.

6. Palumbo V, Boulanger JM, Hill MD, Inzitari D, Buchan AM. Leukoaraiosis and intracerebral hemorrhage after thrombolysis in acute stroke. Neurology. 2007;68:1020-4.
7. Gore JM, Sloan M, Price TR, Randall AM, Bovill E, Collen D, et al. Intracerebral hemorrhage, cerebral infarction, and subdural hematoma after acute myocardial infarction and thrombolytic therapy in the Thrombolysis in Myocardial Infarction Study. Thrombolysis in Myocardial Infarction, Phase II, pilot and clinical trial. Circulation. 1991;83:448-59.

8. Koennecke HC. Cerebral microbleeds on MRI: prevalence, associations, and potential clinical implications. Neurology. 2006;66:165-71.

9. Tsushima Y, Aoki J, Endo K. Brain microhemorrhages detected on T2*-weighted gradient-echo MR images. AJNR Am J Neuroradiol. 2003;24:88-96.

10. Cordonnier C, Al-Shahi Salman R, Wardlaw J. Spontaneous brain microbleeds: systematic review, subgroup analyses and standards for study design and reporting. Brain. 2007;130:19882003.

11. Winkler DT, Biedermann L, Tolnay M, Allegrini PR, Staufenbiel $\mathrm{M}$, Wiessner C, et al. Thrombolysis induces cerebral hemorrhage in a mouse model of cerebral amyloid angiopathy. Ann Neurol. 2002;51:790-3.

12. Montaner J, Molina CA, Monasterio J, Abilleira S, Arenillas JF, Ribo M, et al. Matrix metalloproteinase-9 pretreatment level predicts intracranial hemorrhagic complications after thrombolysis in human stroke. Circulation. 2003;107:598-603. 